\title{
The R-Calculus and the Finite Injury Priority Method
}

\author{
Li Wei ${ }^{1}$, Sui Yuefei ${ }^{2 *}$ \\ ${ }^{1}$ State Key Laboratory of Software Development Environment, Beijing University of Aeronautics and \\ Astronautics, Beijing, China. \\ ${ }^{2}$ Key Laboratory of Intelligent Information Processing, Institute of Computing Technology, Chinese \\ Academy of Sciences, Beijing, China. \\ * Corresponding author. Tel.: (086)-(010)-62600538; email: yfsui@ict.ac.cn \\ Manuscript submitted August 28, 2015; accepted December 28, 2015. \\ doi: $10.17706 /$ jcp.12.2.127-134
}

\begin{abstract}
The R-calculus is a Gentzen-type deduction system to deduce a consistent theory $\Theta$ from a theory $\Gamma$ to be revised and a theory $\Delta$ to revise. Because the semi-decidability of the deduction in the first-order logic, the R-calculus is semi-decidable. By using the limit lemma and finite injury priority method in recursion theory, we shall recursively construct a sequence $\left\{\Theta_{s}: s \in \omega\right\}$ of formula sets such that the limit of $\left\{\Theta_{s}: s \in \omega\right\}$ exists, say $\Theta ; \Delta \mid \Gamma \Rightarrow \Theta$ is provable in the R-calculus, and each formula $\phi$ in $\Gamma$ is enumerated in or extracted from $\Theta$ only finitely often, where $\Theta$ is a maximal consistent set of $\Gamma$ by $\Delta$. Moreover, a Gentzen-type deduction is constructed to deduce the sequence $\left\{\Theta_{s}: s \in \omega\right\}$ by the deduction rules in which the deduction is recursive (decidable, computable).
\end{abstract}

Key words: Belief revision, R-calculus, finite injury priority method, simple set, recursively enumerable sets.

\section{Introduction}

The AGM postulates [1]-[3] are an axiomatized approach for the revision $K \circ \phi$ of a theory $K$ by a formula $\phi$; and the DP postulates [4] are for the iterated revision $\left(\cdots\left(K \circ \phi_{1}\right) \circ \cdots\right) \circ \phi_{n}$, where $\phi$ is a formula and $K$ is a theory in the propositional logic.

The R-calculus [5] gave a Gentzen-type deduction system to deduce a consistent one $\Gamma^{\prime} \cup \Delta$ from an inconsistent theory $\Gamma \cup \Delta$ of the first-order logic, where $\Gamma^{\prime} \cup \Delta$ should be a maximal consistent sub-theory of $\Gamma \cup \Delta ; \Delta \mid \Gamma$ is an R-configuration, $\Gamma$ is a consistent set of formulas, and $\Delta$ is a consistent set of atomic formulas or the negation of atomic formulas. It was proved that if $\Delta|\Gamma \Rightarrow \Delta| \Gamma^{\prime}$ is deducible and $\Delta \mid \Gamma^{\prime}$ is an R-termination, i.e., there is no R-rule to reduce $\Delta \mid \Gamma^{\prime}$ to another R-configuration $\Delta \mid \Gamma^{\prime \prime}$, then $\Delta \cup \Gamma^{\prime}$ is a contraction of $\Gamma$ by $\Delta$.

The finite injury priority method was firstly given by Friedberg [6] and Muchnik [7], who solved the Post problem independently. To construct a recursively enumerable set, the conditions that the set should satisfy are represented by an infinite set of requirements which are decomposed into the positive ones (putting elements in the set) and the negative ones (restraining elements from entering the set). The requirements are ordered by a priority ranking, so that the satisfaction of a requirement may injure the satisfaction of requirements with lower priority and cannot injure the satisfaction of requirements with higher priority [8].

There are correspondences between the R-calculus and the finite injury priority method: 
- In R-calculus, $\Delta \mid \Gamma$ should be consistent. Because the deduction in the first-order logic is semi-decidable, whether $\Delta \cup\{\phi\}$ is consistent can be approximated by a computable decision $\Delta \vdash_{\mathrm{s}} \phi$ (the deduction with length less than s). In recursion theory, the set to be constructed must be recursively enumerable, whether $\{e\}^{A}(x)$ converges can be approximated by a recursive computation $\{e\}_{s}^{A_{s}}(x)$;

- In R-calculus, $\Delta \mid \Gamma$ is a union of a sequence $\left\{\Delta_{i}: i \in \omega\right\}$ of theories, where each $\Delta_{i}$ is approximated by $\Delta_{i, s}$. In recursion theory, the constructed set $\mathrm{A}$ is approximated by $\mathrm{A}_{\mathrm{s}}$, where $\mathrm{A}_{\mathrm{s}}$ is the set of elements entering A before stage s.

Let $\Theta$ be a theory such that $\Delta \mid \Gamma \Rightarrow \Theta$ is provable in the R-calculus. Then, it is proved in [9] that $\Theta$ is a maximal consistent set of $\Gamma$ by $\Delta$, i.e., $\Gamma \cup \Delta \supseteq \Theta \supseteq \Delta$, and for any $\Xi$ with $\Theta \subset \Xi \subseteq \Gamma \cup \Delta, \Xi$ is inconsistent.

In terms of the finite injury priority method, we can recursively construct a sequence $\left\{\Theta_{s}: s \in \omega\right\}$ of theories such that $\lim _{s \rightarrow \infty} \Theta_{s}=\Theta$ exists; $\Delta \mid \Gamma \Rightarrow \Theta$ is provable in R-calculus, and each formula $\phi$ in $\Gamma$ is enumerated in or extracted from $\Theta$ only finitely often.

Therefore, we have the following correspondence:

\begin{tabular}{|l|c|c|}
\hline & First-Order Logic & R-Calculus \\
\hline Semi-decidable process & $\vdash$ & $\Delta \mid \Gamma \Rightarrow \Theta$ \\
\hline Decidable process & $\vdash_{s}$ & $\Delta \mid \Gamma \Rightarrow_{s} \Theta$ \\
\hline
\end{tabular}

The paper is organized as follows: the next section introduces the R-calculus; the third section gives a construction with oracle for the R-calculus; the fourth section defines the approximation deduction in the first-order logic; the fifth section gives a recursive construction for the R-calculus, and the last section concludes the whole paper.

\section{The R-Calculus}

The logical language for the first-order logic contains the following symbols:

variables: $x_{0}, x_{1}, \ldots$;

constants: $c_{0}, c_{1}, \ldots$;

function symbols: $f_{0}, f_{1}, \ldots$;

predicate symbols: $p_{0}, p_{1}, \ldots$;

logical connectives: $\neg, \wedge, \vee, \rightarrow$

and quantifiers: $\forall, \exists$. Terms are defines as

where $f$ is an $n$-ary function symbol.

$$
t::=c|x| f\left(x_{1}, \ldots, x_{n}\right)
$$

Formulas are defined as

$$
\phi=p\left(t_{1}, \ldots, t_{n}\right)|\neg \phi| \phi_{1} \wedge \phi_{2}\left|\phi_{1} \vee \phi_{2}\right| \phi_{1} \rightarrow \phi_{2} \mid \forall x \phi(x),
$$

where $p$ is an $n$-ary predicate symbol, and $t_{1}, \ldots, t_{n}$ are terms.

The R-calculus is defined on a first-order logical language. Let $L$ be a logical language of the first-order logic; $\phi, \psi$ formulas and $\Gamma, \Delta$ sets of formulas (theories).

Given two theories $\Gamma$ and $\Delta$, let $\Delta \mid \Gamma$ be an R-configuration.

The R-calculus $\mathrm{S}$ consists of the following rules:

$$
\begin{gathered}
\left(S^{\text {con }}\right) \frac{\phi \cup \Delta \text { is consistent }}{\Delta|\phi, \Gamma \Rightarrow \Delta, \phi| \Gamma} \\
(S \neg) \frac{\Delta \vdash \neg p}{\Delta|p, \Gamma \Rightarrow \Delta| \Gamma}
\end{gathered}
$$


$\left(\mathrm{S}_{1}^{\wedge}\right) \frac{\Delta\left|\phi_{1}, \Gamma \Rightarrow \Delta\right| \Gamma}{\Delta\left|\phi_{1} \wedge \phi_{2}, \Gamma \Rightarrow \Delta\right| \Gamma}$ if $\Delta \cup\left\{\phi_{1}\right\}$ is inconsistent

$\left(\mathrm{S}_{2}^{\wedge}\right) \frac{\Delta\left[\phi_{1}\right]\left|\phi_{2}, \Gamma \Rightarrow \Delta,\left[\phi_{1}\right]\right| \Gamma}{\Delta\left|\phi_{1} \wedge \phi_{2}, \Gamma \Rightarrow \Delta\right| \Gamma}$ if $\Delta \cup\left\{\phi_{1}\right\}$ is consistent

$\left(S^{\vee}\right) \frac{\Delta\left|\phi_{1}, \Gamma \Rightarrow \Delta\right| \Gamma \Delta\left|\phi_{2}, \Gamma \Rightarrow \Delta\right| \Gamma}{\Delta\left|\phi_{1} \vee \phi_{2}, \Gamma \Rightarrow \Delta\right| \Gamma}$

$\left(S^{\forall}\right) \frac{\Delta|\phi(t), \Gamma \Rightarrow \Delta| \Gamma}{\Delta|\forall x \phi(x), \Gamma \Rightarrow \Delta| \Gamma}$

where $t$ is a term, and $x$ is a new variable not occurring in $\Delta$ and $\Gamma$.

Definition 2.1. $\Delta \mid \Gamma \Rightarrow \Theta$ is provable, denoted by $\vdash_{\mathrm{S}} \Delta \mid \Gamma \Rightarrow \Theta$, if there is a sequence $\left\{\Delta_{1}\left|\Gamma_{1}, \ldots, \Delta_{n}\right| \Gamma_{n}, \ldots\right\}$ of configurations such that $\Delta_{1}\left|\Gamma_{1}=\Delta\right| \Gamma, \Theta=\lim _{n \Rightarrow \infty} \Delta_{n}$, and for each $j, \Delta_{j} \mid \Gamma_{j}$ is deduced from the previous statements by the deduction rules in $\mathbf{S}$.

Theorem 2.2(The soundness theorem[9]). Given a statement $\Delta \mid \Gamma \Rightarrow \Theta$, if $\Delta \mid \Gamma \Rightarrow \Theta$ is provable in the R-calculus then $\Theta$ is a maximal consistent set of $\Gamma$ by $\Delta$.

Theorem 2.3 (The completeness theorem[9]). Given a statement $\Delta \mid \Gamma \Rightarrow \Theta$, if $\Theta$ is a consistent set of $\Gamma$ by $\Delta$ then there is an ordering $\prec$ of $\Gamma$ such that $\Delta \mid \Gamma^{\prec} \Rightarrow \Theta$ is provable in the R-calculus.

\section{The Construction with Oracle}

Fix two consistent theories $\Delta, \Gamma$. We shall construct a theory $\Theta$, satisfying the following conditions:

(3.1) $\Delta \subseteq \Theta \subseteq \Gamma \cup \Delta$;

(3.2) $\Delta$ is a maximal consistent subset of $\Gamma$ by $\Delta$.

Let $\Gamma=\left\{\phi_{0}, \phi_{1}, \ldots\right\}$ and for each $i, \Gamma_{i}=\left\{\phi_{0}, \phi_{1}, \ldots, \phi_{i}\right\}$. We shall construct a sequence $\left\{\Theta_{0}, \Theta_{1}, \ldots\right\}$ of theories such that $\Theta_{0}=\Delta$; for each $i, \Theta_{i+1} \subseteq \Gamma_{i} \cup \Delta$ is a maximal consistent subset of $\Gamma_{i} \cup \Delta$, and $\Theta=\bigcup_{i} \Theta_{i}$ satisfies the above two conditions.

The construction is in stages with oracle whether $\Theta_{i} \cup\left\{\phi_{i}\right\}$ is consistent.

It suffices to meet for each e the following requirements:

$$
P_{e}: \Theta_{e} \cup\left\{\phi_{e}\right\} \text { consistent } \Rightarrow \phi_{e} \in \Theta_{e+1} ;
$$

$N_{e}: \Theta_{e} \cup\left\{\phi_{e}\right\}$ inconsistent $\Rightarrow \phi_{e} \notin \Theta_{e+1}$.

If $\left\{\Theta_{i}: i \in \omega\right\}$ is a sequence satisfying all the requirements then $\Theta=\bigcup_{i} \Theta_{i}$ satisfies (2.1) and (2.2).

The construction:

Stage $s=0$. Define $\Theta_{0}=\Delta$, and for each $i \geq 0, \Theta_{i}=\Delta$.

Stage $s+1$. If $\Theta_{s} \cup\left\{\phi_{s}\right\}$ is consistent then let $\Theta_{s+1}=\Theta_{s} \cup\left\{\phi_{s}\right\}$;

and if $\Theta_{s} \cup\left\{\phi_{s}\right\}$ is inconsistent then let $\Theta_{s+1}=\Theta_{s}$.

This ends the construction.

Lemma 3.1. For each $e, \Theta_{e+1}$ is consistent.

Proof. By induction on $e$. Assume that $\Theta_{e}$ is consistent. Then,

$$
\Theta_{e+1}= \begin{cases}\Theta_{e} \cup\left\{\phi_{e}\right\} \quad \text { if } \Theta_{e} \cup\left\{\phi_{e}\right\} \text { is consistent } \\ \Theta_{e} \text { otherwise }\end{cases}
$$

is consistent.

Lemma 3.2. For each $e, \Theta_{e+1} \subseteq \Delta \cup \Gamma_{\mathrm{e}}$.

Proof. By the construction.

Lemma 3.3. For each $e, \Theta_{e+1}$ is a maximal consistent set of $\Gamma_{e}$ by $\Delta$.

Proof. By the construction, 


$$
\Theta_{e+1}= \begin{cases}\Theta_{e} \cup\left\{\phi_{e}\right\} \quad \text { if } \Theta_{e} \cup\left\{\phi_{e}\right\} \text { is consistent } \\ \Theta_{e} \text { otherwise }\end{cases}
$$

By induction on $e$, if $\Theta_{e}$ is a maximal consistent subset of $\Delta \cup \Gamma_{e-1}$ then $\Theta_{e+1}$ is a maximal consistent subset of $\Delta \cup \Gamma_{e}$.

Therefore, we have that $\Theta$ is a maximal consistent set of $\Gamma$ by $\Delta$.

By the soundness and completeness theorem of R-calculus, we have that $\Theta$ is a maximal consistent set of $\Gamma$ by $\Delta$, and hence, $\Delta \mid \Gamma \Rightarrow \Theta$ is provable in the R-calculus [9].

Also, we can give a deduction form of the construction.

Let $\Gamma_{i, s}$ be the formulas in $\Gamma_{i}=\left\{\phi_{0}, \ldots, \phi_{i-1}\right\}$ which are not deleted at the end of stage $s$ and for each $j<i, \phi_{j} \in \Gamma_{i, s}$ iff $\Delta, \Gamma_{i, s} \sharp \neg \phi_{j}$, and $\Gamma_{i+2, s+1}^{\prime}=\left\{\phi_{i+1}, \phi_{i+2}, \ldots\right\}$.

At stage $s+1$, we consider $\phi_{s}$.

The Gentzen-typed deduction system $\boldsymbol{S}^{\text {app }}$ consists of the following rules:

$$
\begin{aligned}
& \left(\mathbf{S}^{\text {con,app }}\right) \frac{\Delta, \Gamma_{S, s} \forall \neg \phi_{s}}{\Delta, \Gamma_{S, S}\left|\phi_{S,} \Gamma_{S+2, S} \Rightarrow_{s} \Delta, \Gamma_{S, S+1}\right| \Gamma_{S+2, S+1}} ; \\
& (\mathbf{S}\urcorner \text {,app }) \frac{\left.\Delta, \Gamma_{S, s} \vdash\right\urcorner p}{\Delta, \Gamma_{S, S}\left|p, \Gamma_{S+2, S} \Rightarrow \Delta, \Gamma_{S, S+1}\right| \Gamma_{S+2, S+1}^{\prime}} ; \\
& \left(\mathbf{S}_{1}^{\wedge, \text { app }}\right) \frac{\Delta, \Gamma_{S, s}\left|\phi_{s 1}, \Gamma_{S+1, s} \Rightarrow \Delta, \Gamma_{s, s}\right| \Gamma_{S+2, s}^{\prime}}{\Delta, \Gamma_{s, s}\left|\phi_{s 1} \wedge \phi_{s 2}, \Gamma_{s+2, s} \Rightarrow \Delta, \Gamma_{s, s+1}\right| \Gamma_{s+2, s+1}^{\prime}} ;
\end{aligned}
$$

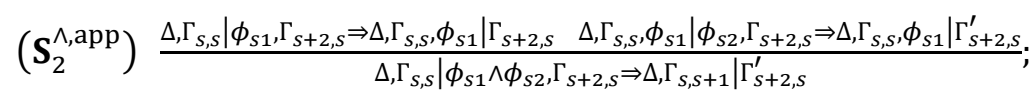

$$
\begin{aligned}
& \left(\mathbf{S}^{\vee, \mathrm{app}}\right) \frac{\Delta, \Gamma_{S, s}\left|\phi_{s 1}, \Gamma_{s+2, s} \Rightarrow \Delta, \Gamma_{S, s}\right| \Gamma_{s+2, s}^{\prime} \quad \Delta, \Gamma_{S, s}\left|\phi_{s 2}, \Gamma_{s+2, s} \Rightarrow \Delta, \Gamma_{S, s}\right| \Gamma_{s+2, s}^{\prime}}{\Delta, \Gamma_{s, s}\left|\phi_{s 1} \vee \phi_{s 2}, \Gamma_{S+2, s} \Rightarrow \Delta, \Gamma_{s, s+1}\right| \Gamma_{s+2, s+1}^{\prime}} ; \\
& \left(\mathbf{S}_{1}^{\forall, \text { app }}\right) \frac{\Delta, \Gamma_{S, s}\left|\phi_{s}(t), \Gamma_{s+1, s} \Rightarrow \Delta, \Gamma_{S, s}\right| \Gamma_{S+2, s}^{\prime}}{\Delta, \Gamma_{S, s}\left|\forall x \phi_{s}(x), \Gamma_{S+2, s} \Rightarrow \Delta, \Gamma_{S, s+1}\right| \Gamma_{s+2, s+1}^{\prime}}
\end{aligned}
$$

where $\Theta_{s}=\Delta \cup \Gamma_{s, s}$, and

$$
\Gamma_{s, S+1}=\left\{\begin{array}{l}
\Gamma_{s, S} \cup\left\{\phi_{s}\right\} \quad \text { if } S=S^{\text {con }} \\
\Gamma_{s, S} \text { otherwise }
\end{array}\right.
$$

By the soundness and completeness theorem ([9]) of the R-calculus $\mathbf{S}$, for any $i \in \omega$, there is a stage $s_{i}$ such that

$$
\Gamma_{i, s_{i}}=\lim _{s \Rightarrow \infty} \Gamma_{i, s_{i}}
$$

and $\Delta \mid \Gamma \Rightarrow \Sigma$ is provable in $\mathbf{S}^{\text {app }}$, where

$$
\Sigma=\Delta \cup \bigcup_{i} \Gamma_{i, s_{i}}
$$

Definition 3.4. $\Delta \mid \Gamma \Rightarrow \Theta$ is provable, denoted by $\vdash s^{\text {app }} \Delta \mid \Gamma \Rightarrow \Theta$, if there is a sequence $\left\{\Delta_{1}\left|\Gamma_{1}, \ldots, \Delta_{n}\right| \Gamma_{n}, \ldots\right\}$ such that $\Delta_{1}\left|\Gamma_{1}=\Delta\right| \Gamma, \Theta=\lim _{n \Rightarrow \infty} \Delta_{n}$, and for each $j<n, \Delta_{j} \mid \Gamma_{j}$ is deduced from the previous statements by the deduction rules in $\mathbf{S}^{\text {app }}$.

Therefore, we have

Theorem 3.5. Given two theories $\Gamma$ and $\Delta, \vdash \vdash_{\mathbf{s}^{\text {app }}} \Delta \mid \Gamma \Rightarrow \Sigma$; and conversely, if $\vdash \vdash_{\mathbf{S}^{\text {app }}} \Delta \mid \Gamma \Rightarrow \Theta$ then $\Theta=\Sigma$.

\section{The Gentzen Deduction System for Approximation Reasoning}

We have the following table: 


\begin{tabular}{|l|c|c|}
\hline & first-order logic & R-calculus \\
\hline Semi-decidable & $\Gamma \vdash \phi$ & $\Delta \mid \phi \Rightarrow \Delta$ \\
\hline Semi-undecidable & $\Gamma \nvdash \phi$ & $\Delta \mid \phi \Rightarrow \Delta, \phi$ \\
\hline
\end{tabular}

If the semi-decidable $\Gamma \vdash \phi$ can be approximated by $\Gamma \vdash_{s} \phi$ and $\Delta \mid \phi \Rightarrow \Delta$ by $\Delta \mid \phi \Rightarrow_{s} \Delta$, then we have the following table:

\begin{tabular}{|l|c|c|}
\hline & first-order logic & R-calculus \\
\hline Monotonic in $s$ & $\Delta \vdash_{s} \phi$ & $\Delta \mid \phi \Rightarrow_{s} \Delta$ \\
\hline Non-monotonic in $s$ & $\Delta \nvdash_{s} \phi$ & $\Delta \mid \phi \Rightarrow_{s} \Delta, \phi$ \\
\hline
\end{tabular}

where $\Delta \vdash_{s} \phi$ is monotonic in $s$, i.e., for any stage $s, \Delta \vdash_{s} \phi$ implies that for any $t \geq s, \Delta \vdash_{t} \phi$; and $\Delta \nvdash_{s} \phi$ is nonmonotonic in $s$, i.e., for any stage $s, \Delta \nvdash_{s} \phi$ does not imply that for any $t \geq s, \Delta \nvdash_{t} \phi$. $\Delta \mid \phi \Rightarrow_{s} \Delta$ is monotonic in $s$, i.e., for any stage $s, \Delta \mid \phi \Rightarrow_{s} \Delta$ implies that for any $t \geq s, \Delta \mid \phi \Rightarrow_{t} \Delta$; and $\Delta \mid \phi \Rightarrow_{s} \Delta$ is non-monotonic in $s$, i.e., for any stage $s, \Delta \mid \phi \Rightarrow_{s} \Delta, \phi$ does not imply that for any $t \geq$ $s, \Delta \mid \phi \Rightarrow_{t} \Delta, \phi$.

The Gentzen deduction system for approximation reasoning:

- Axiom:

$$
\Gamma, \phi \Rightarrow_{0} \phi, \Delta \quad(I)
$$

- Logical rules:

\begin{tabular}{|c|c|c|c|}
\hline$\frac{\Gamma, \phi \Rightarrow_{s} \Delta}{\Gamma, \phi \wedge \psi \Rightarrow_{s+1} \Delta}$ & $\left(\wedge^{L_{1}}\right)$ & $\frac{\Gamma \Rightarrow_{s} \phi, \Delta \quad \Sigma \Rightarrow_{s} \psi, \Pi}{\Gamma, \Sigma \Rightarrow_{s+1} \phi \wedge \psi, \Delta, \Pi}$ & $\left(\wedge^{R}\right)$ \\
\hline$\frac{\Gamma, \psi \Rightarrow_{s} \Delta}{\Gamma, \phi \wedge \psi \Rightarrow_{s+1} \Delta}$ & $\left(\wedge^{L_{2}}\right)$ & & $\left(\vee^{R_{1}}\right)$ \\
\hline$\frac{\Gamma, \phi \Rightarrow_{s} \Delta \Sigma, \psi \Rightarrow_{s} \Pi}{\Gamma, \Sigma, \phi \vee \psi \Rightarrow_{s+1} \Delta, \Pi}$ & $\left(\vee^{L}\right)$ & $\frac{\Gamma \Rightarrow_{s} \phi, \Delta}{\Gamma \Rightarrow_{s+1} \phi \vee \psi, \Delta}$ & $\left(\vee^{R_{2}}\right)$ \\
\hline$\frac{\Gamma \Rightarrow_{s} \psi, \Delta}{\Gamma \Rightarrow_{s+1} \phi \vee \psi, \Delta}$ & $\frac{\Gamma, \phi \Rightarrow_{s} \Delta}{\Gamma \Rightarrow_{s+1} \neg \phi, \Delta}$ & $\left(\neg^{R}\right)$ \\
\hline$\frac{\Gamma, \phi(t) \Rightarrow_{s} \Delta}{\Gamma, \forall x \phi(x) \Rightarrow_{s+1} \Delta}$ & $\left(\neg^{L}\right)$ & $\frac{\Gamma \Rightarrow_{s} \phi(x), \Delta}{\Gamma \Rightarrow_{s+1} \forall x \phi(x), \Delta}$ & $\left(\forall^{R}\right)$ \\
\hline
\end{tabular}

where in the $\forall$-rules, $t$ is an arbitrary term, and $x$ does not occur in the lower sequent.

Definition 4.1. A sequent $\Gamma \Rightarrow \Delta$ is s-deducible, denoted by $\vdash_{s} \Gamma \Rightarrow \Delta$, if there is a sequence $\Gamma \Rightarrow_{i_{0}} \Delta, \ldots, \Gamma_{n} \Rightarrow_{i_{n}} \Delta_{n}$ which is a proof and $\Gamma_{n}=\Gamma, \Delta_{n}=\Delta$, and for each $i \leq n, i_{n} \leq s$.

Intuitively, a sequent $\Gamma \Rightarrow \Delta$ is s-deducible if there is a deduction tree for $\Gamma \Rightarrow \Delta$ with depth $\leq s$.

Proposition 4.2. (i) For any sequent $\Gamma \Rightarrow \Delta$, if $\vdash_{s} \Gamma \Rightarrow \Delta$ then $\vdash \Gamma \Rightarrow \Delta$.

(ii) For any sequent $\Gamma \Rightarrow \Delta$, if $\vdash \Gamma \Rightarrow \Delta$ then there is an $s \in \omega$ such that $\vdash_{s} \Gamma \Rightarrow \Delta$.

Therefore, $\vdash$ is the limit of $\left\{\vdash_{s}: s \in \omega\right\}$, i.e., $\vdash=\lim _{s \Rightarrow \infty} \vdash_{s}$.

Definition 4.3. A theory $\Gamma$ is $s$-consistent if there is no formula $\phi$ such that $\Gamma \vdash_{s} \phi$ and $\Gamma \vdash_{s} \neg \phi$.

\section{The Recursive Construction}

Let $\Gamma=\left\{\phi_{0}, \phi_{1}, \ldots\right\}$ and for each $i, \Gamma_{i}=\left\{\phi_{0}, \phi_{1}, \ldots, \phi_{i-1}\right\}$. We shall construct in stages a sequence $\left\{\Theta_{0}, \Theta_{1}, \ldots\right\}$ of theories such that $\Theta_{0}=\Delta$; for each $i, \Theta_{i+1} \subseteq \Gamma_{i} \cup \Delta$ is a maximal consistent subset of $\Gamma_{i} \cup \Delta$, and $\Theta=\cup_{i} \Theta_{i}$ satisfies (3.1) and (3.2).

The construction is in stages with approximation deduction $\Theta_{i, s} \vdash_{s} \neg \phi_{i}$, where $\Gamma \vdash_{s} \phi$ if there is a sequence $\left\{\theta_{0}, \ldots, \theta_{n}\right\}$ of formulas with $n \leq s$ which is a proof of $\Gamma \vdash \phi$. 
It suffices to meet for each $e$ the following requirements:

$P_{e}: \Theta_{e} \cup\left\{\phi_{e}\right\}$ consistent $\Rightarrow \phi_{e} \in \Theta_{e+1}$;

$N_{e}: \Theta_{e} \cup\left\{\phi_{e}\right\}$ inconsistent $\Rightarrow \phi_{e} \notin \Theta_{e+1}$.

The priority ranking of requirements are

$$
P_{0}, N_{0}, P_{1}, N_{1}, \ldots, P_{e}, N_{e}, \ldots
$$

If $\left\{\Theta_{i}: i \in \omega\right\}$ is a sequence satisfying all the requirements then $\Theta=\cup_{i} \Theta_{i}$ satisfies (3.1) and (3.2).

A requirement $N_{e}$ requires attention at stage $s+1$ if $\Theta_{e, s} \vdash_{s+1} \neg \phi_{e}$ and $\phi_{e} \in \Theta_{e+1, s}$.

A requirement $N_{e}$ is satisfied at stage $s+1$ if $\Theta_{e, s} \vdash_{s+1} \neg \phi_{e}$ and $\phi_{e} \notin \Theta_{e+1, s}$.

\section{The construction:}

Stage $s=0$. Define $\Theta_{0,0}=\Delta$, and for each $i \geq 0, \Theta_{i, 0}=\Delta \cup \Gamma_{i}$.

Stage $s+1$. Find the least e such that $N_{e}$ requires attention. Define

$\Theta_{e+1, s+1}=\Theta_{e+1, s}-\left\{\phi_{e}\right\}$, and for each $e^{\prime} \geq e+1$,

$$
\Theta_{e^{\prime}+1, s+1}=\Theta_{e^{\prime}, s+1} \cup\left\{\phi_{e^{\prime}}\right\},
$$

and we say that $N_{e}$ receives attention.

Define for each $e$,

$$
\Theta_{e}=\lim _{s \Rightarrow \infty} \Theta_{e, s}
$$

This ends the construction.

We say that $N_{e}$ is injured at stage $s+1$ if there is $i<e$ such that $\phi_{i} \in \Theta_{i+1, s}-\Theta_{i+1, s+1}$. Define the injury set for $N_{e}$ as follows:

$$
\begin{gathered}
I_{e}=\left\{i: \exists s\left(\phi_{i} \in \Theta_{i+1, s}-\Theta_{i+1, s+1}\right)\right\} \\
I_{e, s}=\left\{i: \exists s^{\prime} \leq s\left(\phi_{i} \in \Theta_{i+1, s^{\prime}}-\Theta_{i+1, s^{\prime}+1}\right)\right\} .
\end{gathered}
$$

Lemma 5.1. For each $e, I_{e}$ is finite.

Proof. By induction on $e$. Assume that $I_{e^{\prime}}\left(e^{\prime}<e\right)$ is finite. Then, there is a stage $s_{e}$ such that for any $s \geq s_{e}, N_{e^{\prime}}$ never requires attention, and $I_{e}=I_{e, s_{e}}$ is finite.

Lemma 5.2. For every $e, \Theta_{e}=\lim _{s \Rightarrow \infty} \Theta_{e, s+1}$ exists, and requirement $N_{e}$ is met.

Proof. Fix $e$. By Lemma 3.1, there is a stage $s_{e}$ such that $N_{e}$ is not injured at any stage $s>s_{e}$.

If $N_{e}$ never requires attention then $\Theta_{e}=\Theta_{e, s_{e}}$;

If $N_{e}$ requires attention at some stage $s>s_{e}$ then, $N_{e}$ is satisfied at $s$ and never requires attention. Hence, $\Theta_{e}=\Theta_{e, s}$.

Lemma 5.3. For every $e$, requirement $P_{e}$ is met.

Proof. If $N_{e}$ is satisfied at stage s then so is $P_{e}$.

By the soundness and completeness theorem of R-calculus, we have that $\Theta$ is a maximal consistent set of $\Gamma$ by $\Delta$, and hence, $\Delta \mid \Gamma \Rightarrow \Theta$ is provable in the R-calculus ([9]).

Let $\Gamma_{i, s}$ be the formulas in $\Gamma_{i}=\left\{\phi_{0}, \ldots, \phi_{i-1}\right\}$ which are not deleted at the end of stage $\mathrm{s}$ and for each $j<i, \phi_{j} \in \Gamma_{i, s}$ iff $\Delta, \Gamma_{i, s} \sharp \neg \phi_{j}$.

The approximation $\mathbf{R}$-calculus $\mathbf{S}^{\text {rec }}$ consists of the following two sets of rules: one set is not to eliminate formulas in $\Gamma$, and another is to eliminate.

$$
\begin{gathered}
\left(\mathrm{S}^{\text {con,rec }}\right) \frac{\Delta, \Gamma_{i, s} t_{s} \neg p}{\Delta, \Gamma_{i, s}\left|p, \Gamma_{i+2, s} \Rightarrow i \Delta, \Gamma_{i, s}, p\right| \Gamma_{i+2, s+1}} ; \\
\left(\mathrm{S}_{\wedge, \text { rec }}\right) \frac{\Delta, \Gamma_{i, s}\left|\phi_{i 1}, \Gamma_{i+2, s} \Rightarrow \Delta, \Gamma_{i, s}, \phi_{i 1}\right| \Gamma_{i+2, s} \Delta, \Gamma_{i, s}\left|\phi_{i 2}, \Gamma_{i+2, s} \Rightarrow \Delta, \Gamma_{i, s}, \phi_{i 2}\right| \Gamma_{i+2, s}}{\Delta, \Gamma_{i, s}\left|\phi_{i 1} \wedge \phi_{i 2}, \Gamma_{i+2, s} \Rightarrow \Delta, \Gamma_{i, s+1}\right| \Gamma_{i+2, s+1}} ;
\end{gathered}
$$




$$
\begin{aligned}
& \left(\mathrm{S}_{\mathrm{V}, \mathrm{rec}}^{1}\right) \frac{\Delta, \Gamma_{i, s}\left|\phi_{i 1}, \Gamma_{i+2, s} \Rightarrow \Delta, \Gamma_{i, s}, \phi_{i 1}\right| \Gamma_{i+2, s}}{\Delta, \Gamma_{i, s}\left|\phi_{i 1} \vee \phi_{i 2}, \Gamma_{i+2, s} \Rightarrow \Delta, \Gamma_{i, s+1}\right| \Gamma_{i+2, s+1}} ; \\
& \left(\mathrm{S}_{\mathrm{V}, \mathrm{rec}}^{2}\right) \frac{\Delta, \Gamma_{i, s}\left|\phi_{i 2}, \Gamma_{i+2, s} \Rightarrow \Delta, \Gamma_{i, s}, \phi_{i 2}\right| \Gamma_{i+2, s}}{\Delta, \Gamma_{i, s}\left|\phi_{i 1} \vee \phi_{i 2}, \Gamma_{i+2, s} \Rightarrow \Delta, \Gamma_{i, s+1}\right| \Gamma_{i+2, s+1}} ; \\
& \left(\mathrm{S}_{\forall, \text { rec }}\right) \frac{\Delta, \Gamma_{i, s}\left|\phi_{i}(t), \Gamma_{i+2, s} \Rightarrow \Delta, \Gamma_{i, s}, \phi_{i}(t)\right| \Gamma_{i+2, s}}{\Delta, \Gamma_{i, s}\left|\forall x \phi_{i}(x), \Gamma_{i+2, s} \Rightarrow \Delta, \Gamma_{i, s+1}\right| \Gamma_{i+2, s+1} ;}
\end{aligned}
$$

where $\Gamma_{i, s+1}=\Gamma_{i, s} \cup\left\{\phi_{i}\right\}, \phi_{i}=p|\neg p| \phi_{i 1} \wedge \phi_{i 2}\left|\phi_{i 1} \vee \phi_{i 2}\right| \phi_{i 1} \Rightarrow \phi_{i 2}\left|\forall x \phi_{i}(x)\right| \exists x \phi_{i}(x)$, and

$$
\begin{aligned}
& (\mathrm{S} \neg, \mathrm{rec}) \frac{\Delta, \Gamma_{i, s} \vdash_{s} \neg p}{\Delta, \Gamma_{i, s}\left|p, \Gamma_{i+2, s}=\Delta, \Gamma_{i, s+1}\right| \Gamma_{i+2, s+1}^{\prime}} ; \\
& \left(\mathrm{S}_{1}^{\wedge, \mathrm{rec}}\right) \frac{\Delta, \Gamma_{i, s}\left|\phi_{i 1}, \Gamma_{i+1, s} \Rightarrow \Delta, \Gamma_{i, s}\right| \Gamma_{i+2, s}^{\prime}}{\Delta, \Gamma_{i}, s\left|\phi_{i 1} \wedge \phi_{i 2}, \Gamma_{i+2, s} \Rightarrow \Delta, \Gamma_{i, s+1}\right| \Gamma_{i+2, s+1}^{\prime}} ; \\
& \left(\mathrm{S}_{2}^{\wedge, \mathrm{rec}}\right) \frac{\Delta, \Gamma_{i, s}\left|\phi_{i 1}, \Gamma_{i+2, s} \Rightarrow \Delta, \Gamma_{i, s}, \phi_{i 1}\right| \Gamma_{i+2, s} \quad \Delta, \Gamma_{i, s}, \phi_{i 1}\left|\phi_{i 2}, \Gamma_{i+2, s} \Rightarrow \Delta, \Gamma_{i, s}, \phi_{i 1}\right| \Gamma_{i+2, s}^{\prime}}{\Delta, \Gamma_{i, s}\left|\phi_{i 1} \wedge \phi_{i 2}, \Gamma_{i+2, s} \Rightarrow \Delta, \Gamma_{i, s+1}\right| \Gamma_{i+2, s}^{\prime}} ; \\
& \left(S^{\vee, r e c}\right) \frac{\Delta, \Gamma_{i, s}\left|\phi_{i 1}, \Gamma_{i+2, s} \Rightarrow \Delta, \Gamma_{i, s}\right| \Gamma_{i+2, s}^{\prime} \quad \Delta, \Gamma_{i, s}\left|\phi_{i 2}, \Gamma_{i+2, s} \Rightarrow \Delta, \Gamma_{i, s}\right| \Gamma_{i+2, s}^{\prime}}{\Delta, \Gamma_{i, s}\left|\phi_{i 1} \vee \phi_{i 2}, \Gamma_{i+2, s} \Rightarrow \Delta, \Gamma_{i, s+1}\right| \Gamma_{i+2, s+1}^{\prime}} ; \\
& \left(S^{\forall, \text { rec }}\right) \frac{\Delta, \Gamma_{i, s}\left|\phi_{i}(t), \Gamma_{i+1, s} \Rightarrow \Delta, \Gamma_{i, s}\right| \Gamma_{i+2, s}^{\prime}}{\Delta, \Gamma_{i, s}\left|\forall x \phi_{i}(x), \Gamma_{i+2, s} \Rightarrow \Delta, \Gamma_{i, s+1}\right| \Gamma_{i+2, s+1}^{\prime}} ;
\end{aligned}
$$

where $\Delta, \Gamma_{i, s} \mid \phi, \Gamma_{i+2, s}$ means that $\Delta, \Gamma_{i, s}$ is $s$-consistent; and

$$
\Gamma_{i, s+1}=\left\{\begin{array}{l}
\Gamma_{i, s} \cup\left\{\phi_{i}\right\} \quad \text { if } \Gamma_{i, s} \not \neg \phi_{i} \\
\Gamma_{i, s} \text { otherwise }
\end{array}\right.
$$

By the soundness and completeness theorem ([9]) of the R-calculus S, for any $i \in \omega$, there is a stage $s_{i}$ such that

and $\Delta \mid \Gamma \Rightarrow \Sigma$ is provable in $\mathbf{S}^{\text {rec }}$, where

$$
\Gamma_{i, s_{i}}=\lim _{s \Rightarrow \infty} \Gamma_{i, s}
$$

$$
\Sigma=\Delta \cup \bigcup_{i} \Gamma_{i, s_{i}}
$$

Definition 5.4. $\Delta \mid \Gamma \Rightarrow \Theta$ is provable, denoted by $\vdash_{S^{r}}$ rec $\Delta \mid \Gamma \Rightarrow \Theta$, if there is a sequence $\left\{\Delta_{1}\left|\Gamma_{1}, \ldots, \Delta_{n}\right| \Gamma_{n}, \ldots\right\}$ such that $\Delta_{1}\left|\Gamma_{1}=\Delta\right| \Gamma, \Theta=\lim _{n \rightarrow \infty} \Delta_{n}$, and for each $j<n, \Delta_{j} \mid \Gamma_{j}$ is deduced from the previous statements by the deduction rules in $\mathbf{S}^{\text {rec }}$.

Therefore, we have

Theorem 5.5. Given two theories $\Gamma$ and $\Delta, \vdash_{S^{\text {rec }}} \Delta \mid \Gamma \Rightarrow \Sigma$; and conversely, if $\vdash_{S^{\text {rec }}} \Delta \mid \Gamma \Rightarrow \Theta$ then $\Theta=\Sigma$.

\section{Conclusion}

By using the finite injury priority method in recursion theory, we gave a recursive construction of $\Theta$ such that $\Delta \mid \Gamma \Rightarrow \Theta$ is provable in the R-calculus, which makes recursive the construction with oracle, with the cost of finite injuries (some formulas in $\Gamma$ may be enumerated in $\Gamma$ or extracted from $\Gamma$ finitely often).

\section{Acknowledgment}

This work was supported by the Open Fund of the State Key Laboratory of Software Development Environment under Grant No. SKLSDE-2010KF-06, Beijing University of Aeronautics and Astronautics, and by the National Basic Research Program of China (973 Program) under Grant No.2005CB321901.

\section{References}


[1] Alchourrón, C. E., Gärdenfors, P., \& Makinson, D. (1985). On the logic of theory change: Partial meet contraction and revision functions. J. Symbolic Logic, 50, 510-530.

[2] Fermé, E., \& Hansson, S. 0. (2011). AGM 25 years, twenty-five years of research in belief change. Journal of Philosophical Logic, 40, 295-331.

[3] Friedman, N., \& Halpern, J. Y. (1996). Belief revision: A critique. Proceedings of 5th Conference on Principles of Knowledge Representation and Reasoning (pp. 421-431).

[4] Darwiche, A., \& Pearl, J. (1997). On the logic of iterated belief revision. Artificial Intelligence, 89, 1-29.

[5] Li, W. (2007). R-calculus: An inference system for belief revision. The Computer J., 50, 378-390.

[6] Friedberg, R. M. (1957). Two recursively enumerable sets of incomparable degrees of unsolvability. Proc. Natl. Acad. Sci., 43, 236-238

[7] Muchnik, A. A. (1956). On the separability of recursively enumerable sets (in Russian). Dokl. Akad. Nauk SSSR, N.S., 109, 29-32.

[8] Rogers, H. (1967). Theory of Recursive Functions and Effective Computability, The MIT Press.

[9] Li, W., Sui, Y., \& Sun, M. (2015). The sound and complete R-Calculus for revising propositional theories. Science China: Information Sciences, 58, 092101:1-092101:12.

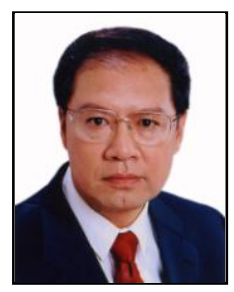

Li Wei is a Professor in the School of Computer Science and Engineering, Beihang University and is a member of the Chinese Academy of Sciences. Li is mostly engaged in the applied research of computer software and theory, and the internet, including programming languages, software development, artificial intelligence, and integrated circuit design.

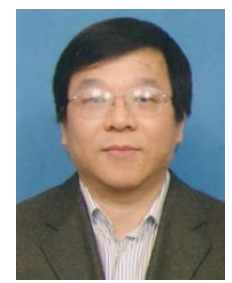

Sui Yuefei is a Professor in the Institute of Computing Technology, Chinese Academy of Sciences. His main interests include knowledge representation, applied logic and the theory of computability. 